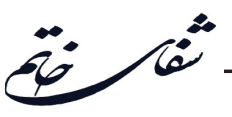

\title{
The Relationship Between Pupil Diameter Data and Confidence in Multi-Stage Decisions
}

\section{Shirin Vafaei ${ }^{1}$, Reza Ebrahimpour ${ }^{1,2 *}$, Sajjad Zabbah ${ }^{2}$}

${ }^{1}$ Faculty of Computer Engineering, Shahid Rajaee Teacher Training University, Tehran, Iran

${ }^{2}$ School of Cognitive Sciences, Institute for Research in Fundamental Sciences (IPM), Tehran, Iran

\section{Article Info:}

\section{ABSTRACT}

Introduction: The basic human decision involves several stages of consecutive decisions. In the presence of multi-stage decisions, to avoid interruption in the sequence of decisions, there is no possibility to request a confidence report on the middle stages. This inevitably makes to estimate the confidence of the decision. The challenges involved in applying behavioral data to determine confidence, make it more important to use other types of data. Since eye data is associated with the decision process, this study examined the dynamics of involuntary information, in the context of multi-stage decisions. The pupil size was applied as a valuable source to estimate the decision confidence. Material and Methods: A two-stage psychophysical experiment was designed. In this experiment, participants were instructed to decide about the direction of moving dots in two successive stimuli (right or left for the first stage and up or down for the second stage). Participants were received correct feedback in a trial when they reported the correct direction of both stimuli. During the experiment, the eye movement data of the participants were recorded by an eye-tracking device. Results: The latency of the pupil diameter signal positive peak and pupil diameter at the time of response were meaningfully associated with decision confidence. Furthermore, the results demonstrated that pupil size was altered in the current decision based on the confidence of the previous decision. Conclusion: This research establishes that in the multis tage decision making, the confidence of a decision is represented in involuntary information that can reflect the individual's strategy in complex situations. In addition, the confidence of

\section{Key words:}

1. Reaction Time

2. Humans

3. Pupil the previous decision can significantly change voluntary and involuntary behaviors in the same manner.

*Corresponding Author: Reza Ebrahimpour

E-mail: rebrahimpour@srttu.edu 


\title{
ارتباط بين دادههاى جشمى مردمك و قطعيت در تصميمهاى جند مرحلهاى
}

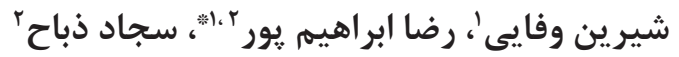 \\ 'دانشكده مهندسى كامبيوتر، دانشعاه تربيت دبير شهيد رجايى، تهران، ايران \\ 'يزوهشكده علوم شناختى، يزوهشعاه دانشهاى بنيادى، تهران، ايران
}

\section{اطلاعات مقاله:}

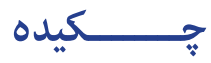

مقدمص: تصميمهاى اساسى انسان شامل جندين مرحله از تصميمهاى متوالى است. در اين گونه تصميمات

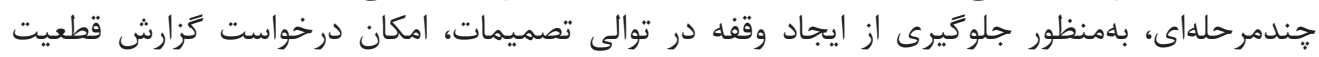

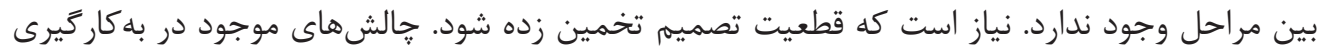

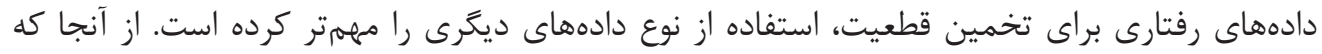

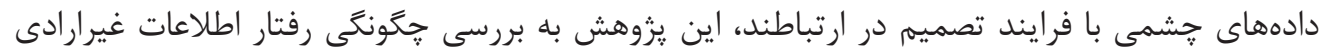

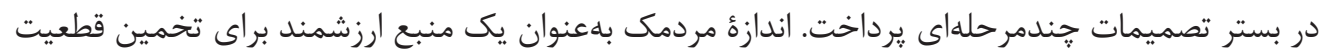

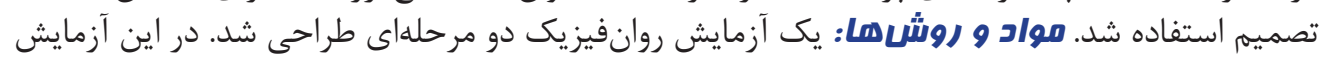
شركت كنندهها در مورد جهت حركت نقاط در دو محرك متوالى (راست يا جِ

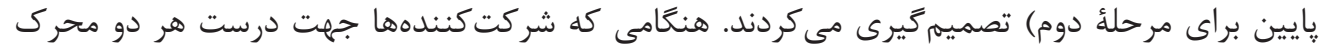

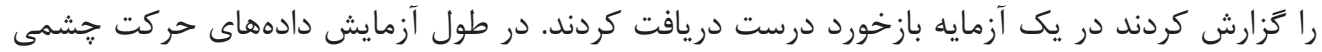

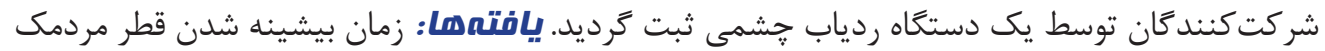

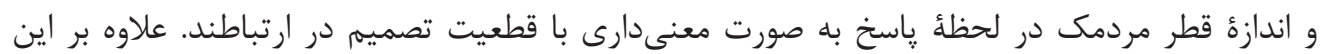

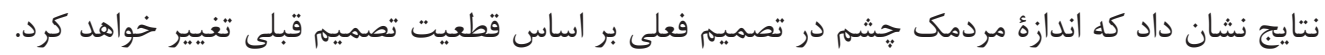

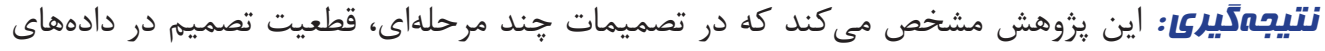

كليد وازهها:

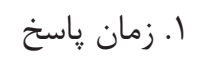

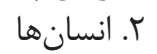

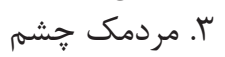

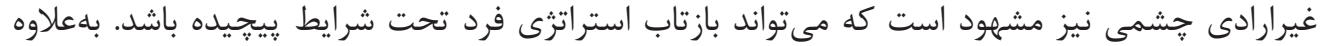

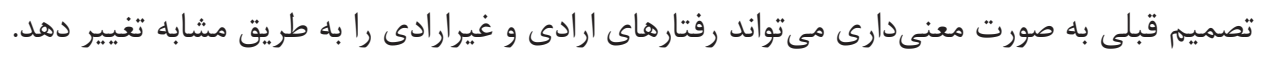

* نويسنده مسئول: رضا ابراهيم :ور آدرس الكترونيكى: rebrahimpour@srttu.edu 


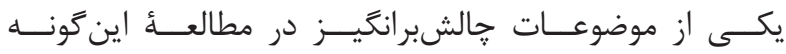

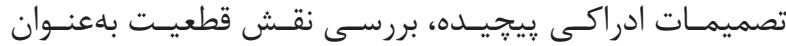

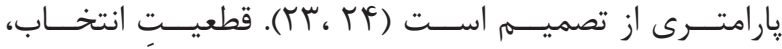

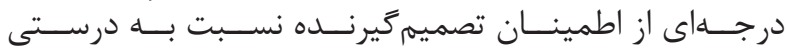

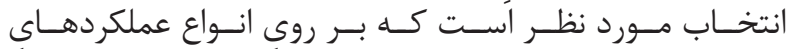

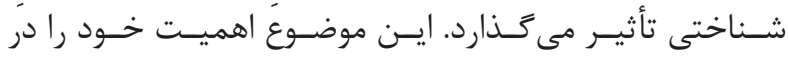

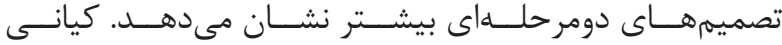

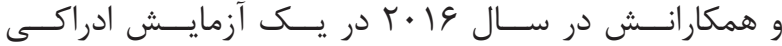

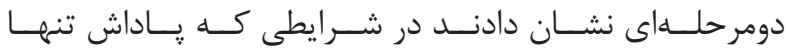

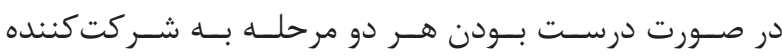

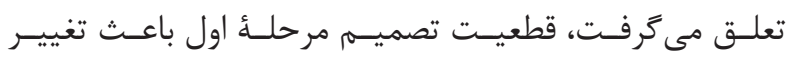

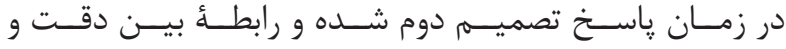

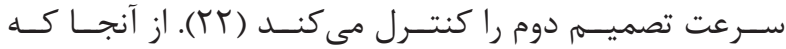

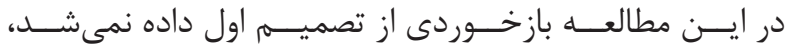

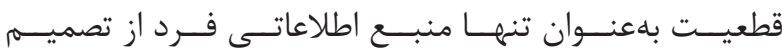

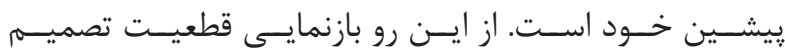

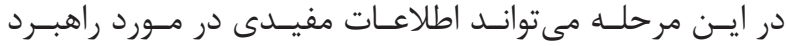

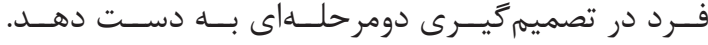

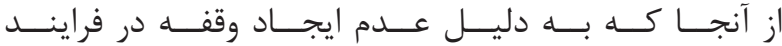

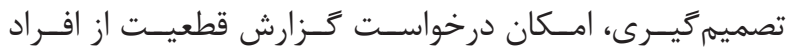

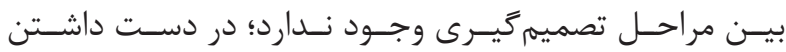

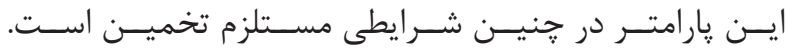

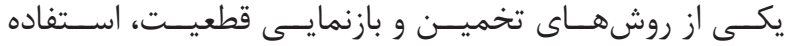

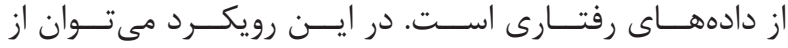

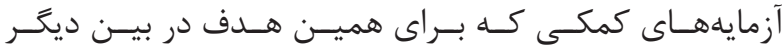

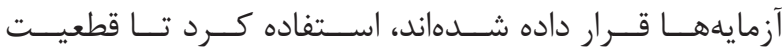

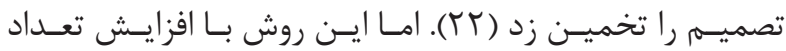

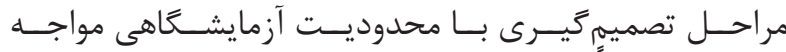

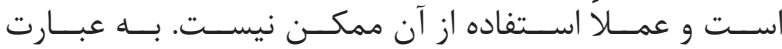

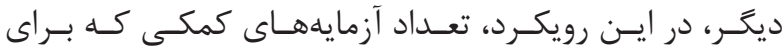

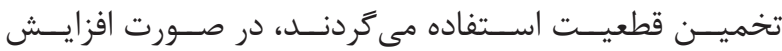

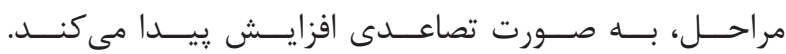

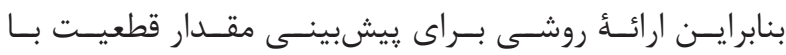

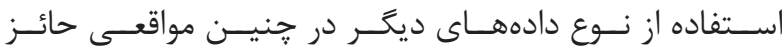

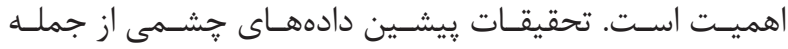

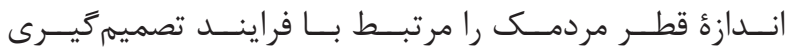

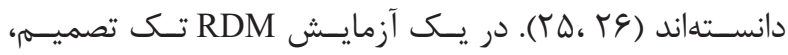

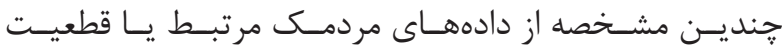

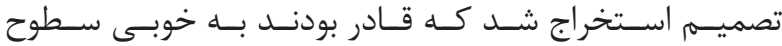

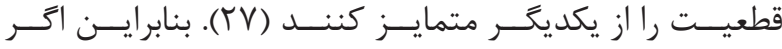

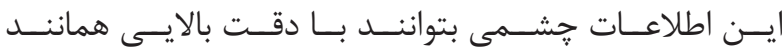

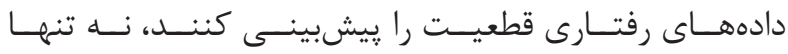

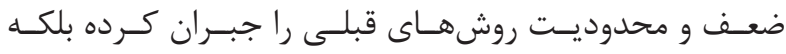

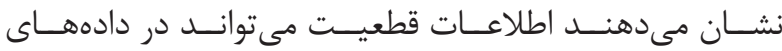

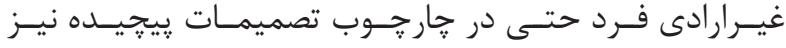

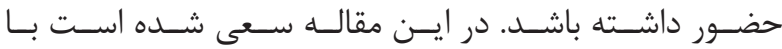

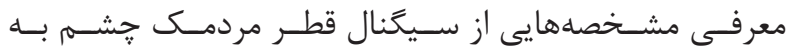

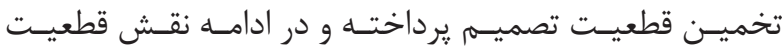

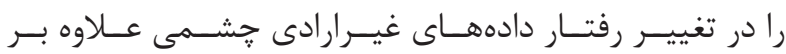

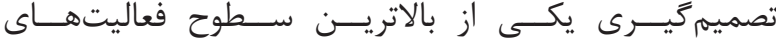

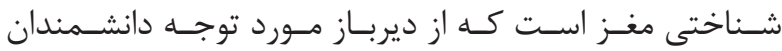

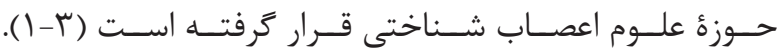

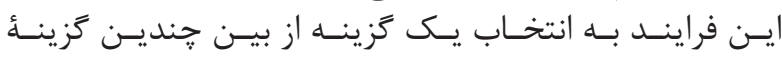

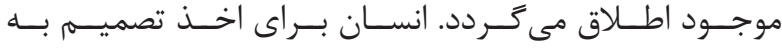

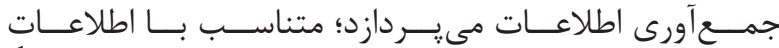

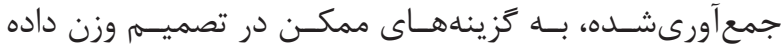

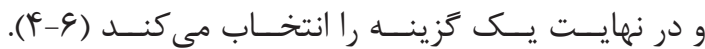

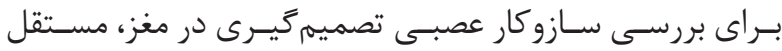

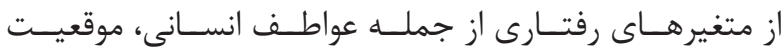

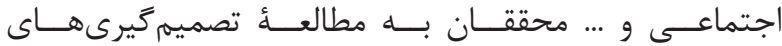

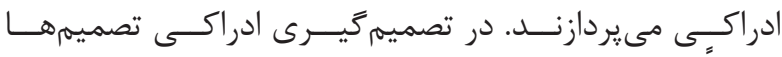

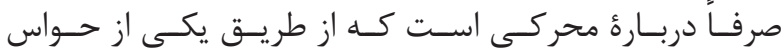

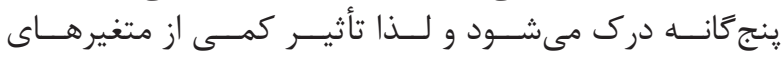

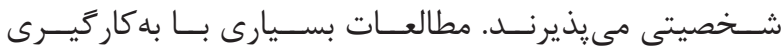

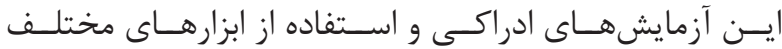

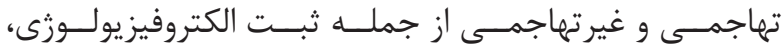

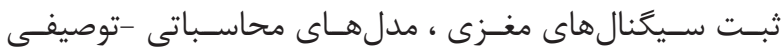

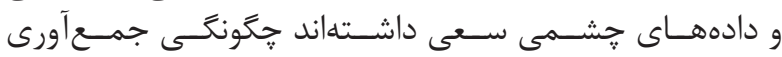

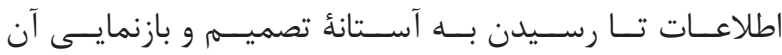

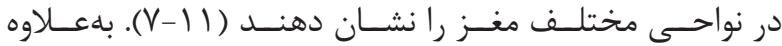

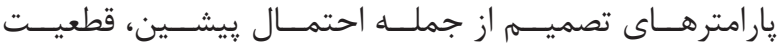

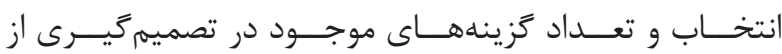

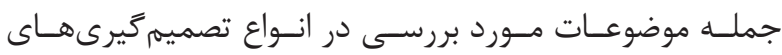

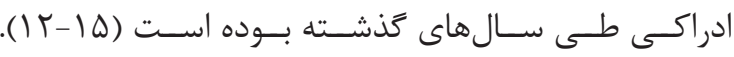

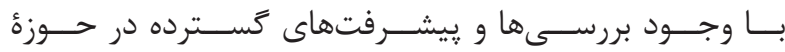

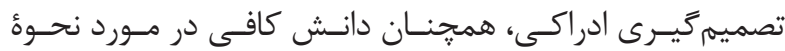

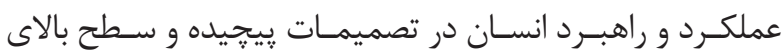

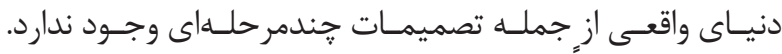

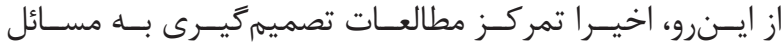

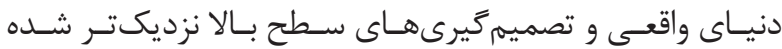

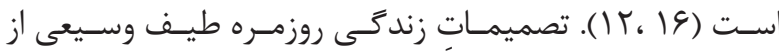

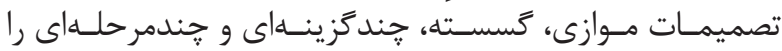

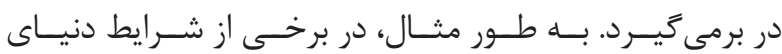

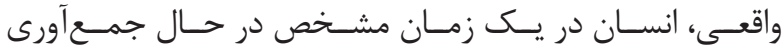

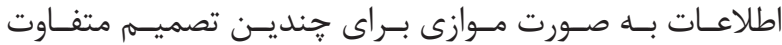

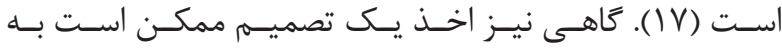

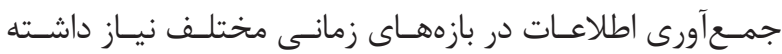

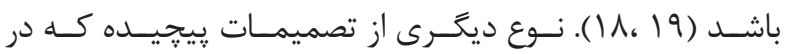

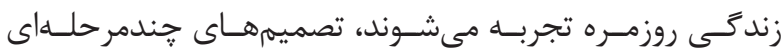

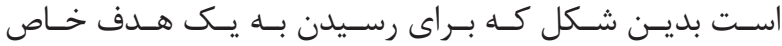

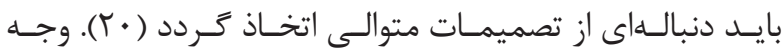

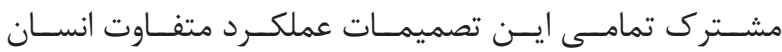

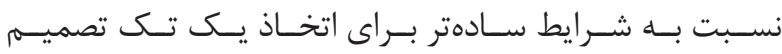

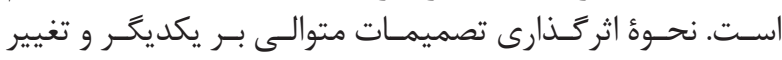

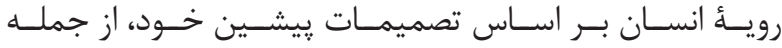

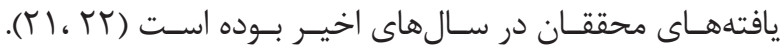




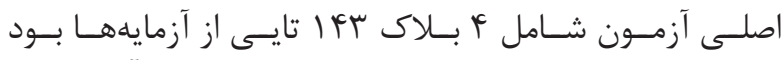

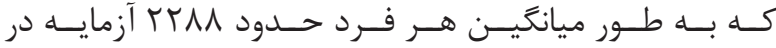

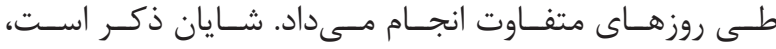

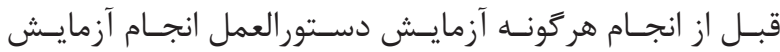

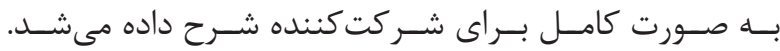

محرك بينايى

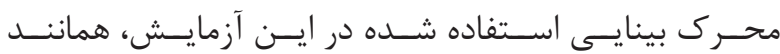

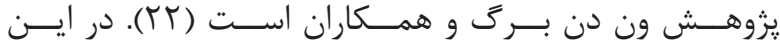

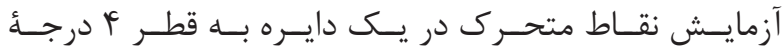

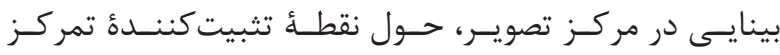

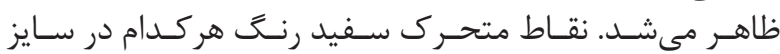

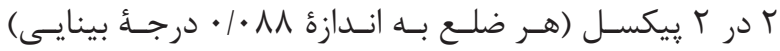

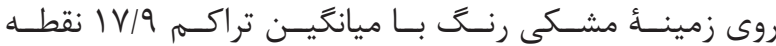

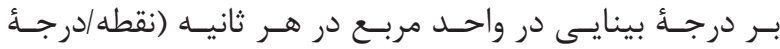

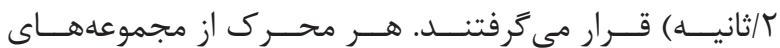

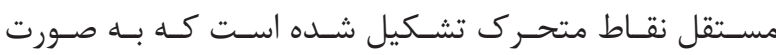

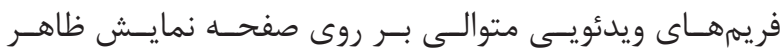
مى شـــند

$$
\text { طراحى آزمايش }
$$

آزمايـش در يـك اتـاق نيمهتاريـــ و ايزولـهـ شــده در برابـر

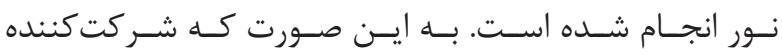

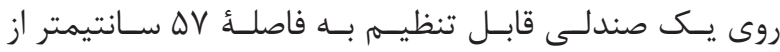

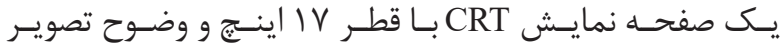

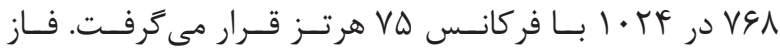

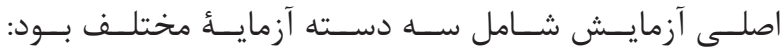

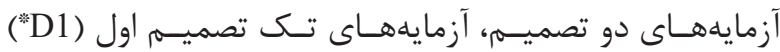

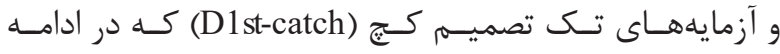

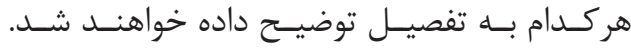

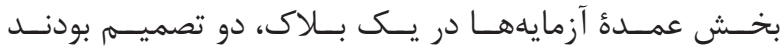

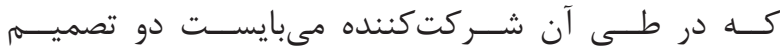

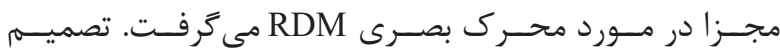

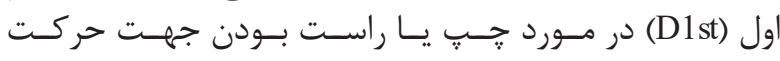

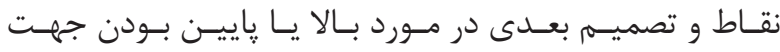

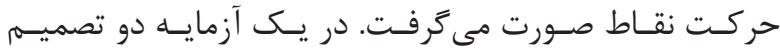

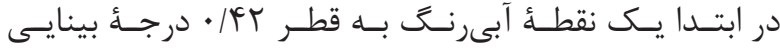

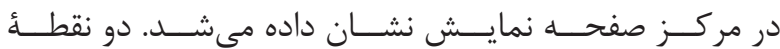

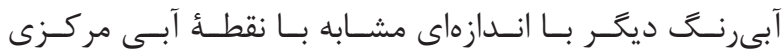

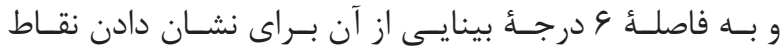

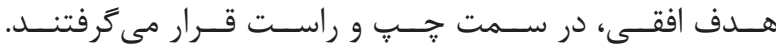

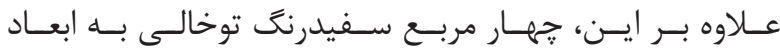

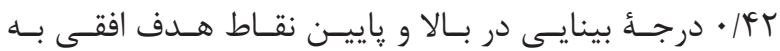

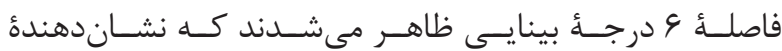

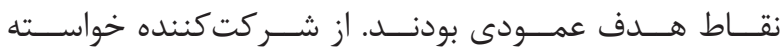

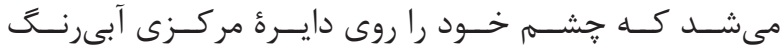

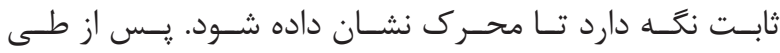

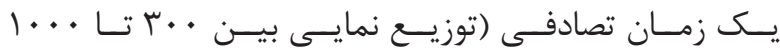

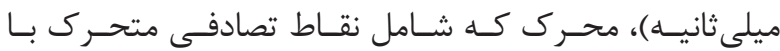

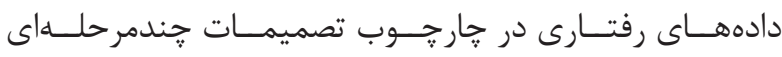
نشـان دهيسم.

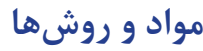

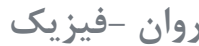

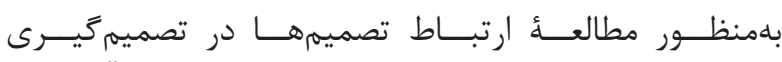

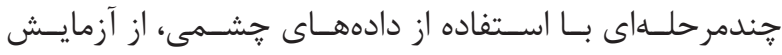

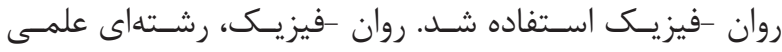

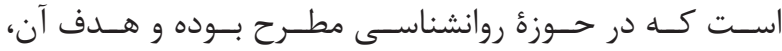

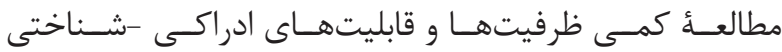

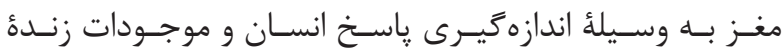

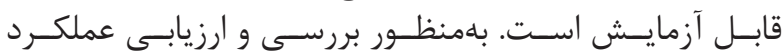

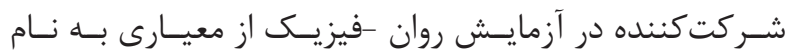

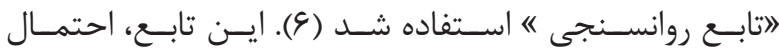

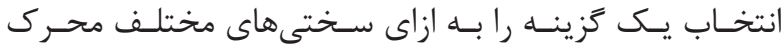

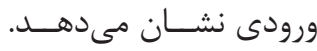
دستخاه ردياب ״شمى

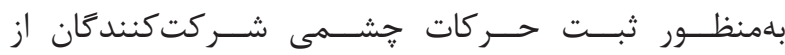

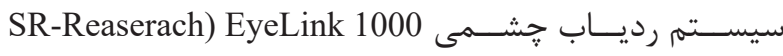

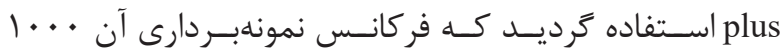

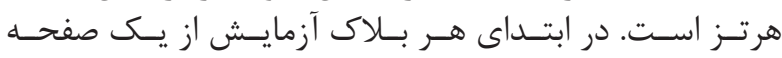

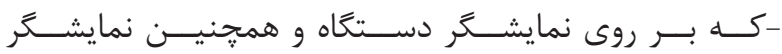

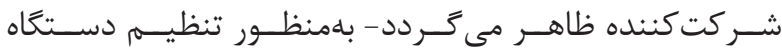

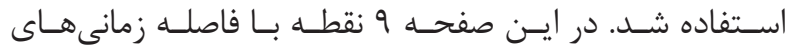

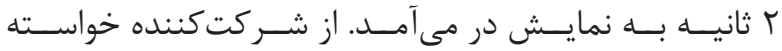

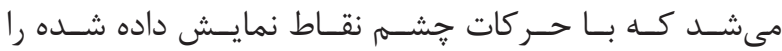

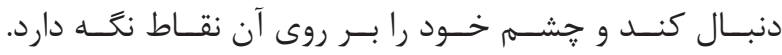

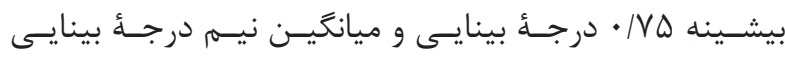

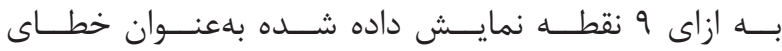

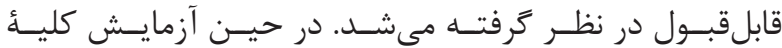

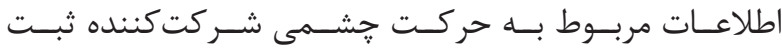

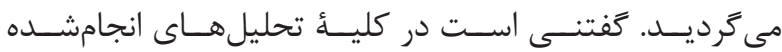

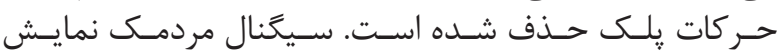

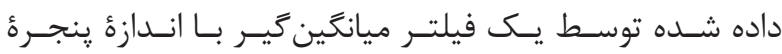

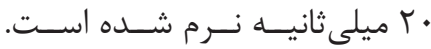

شر كت كنندهها

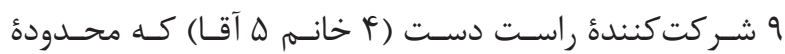

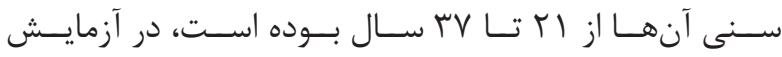

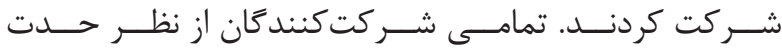

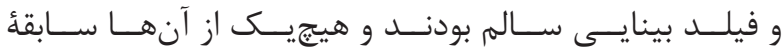

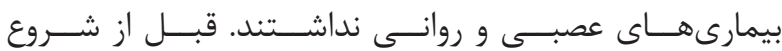

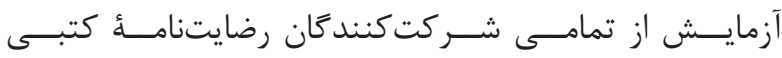

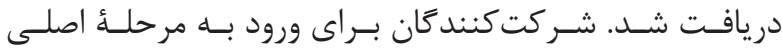

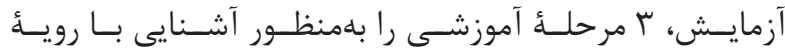

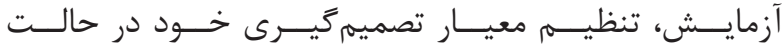

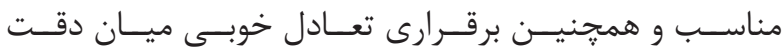

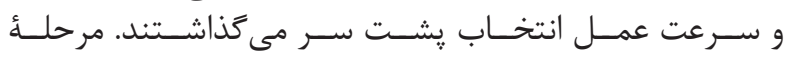




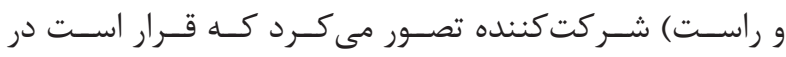

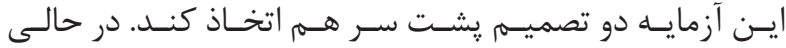

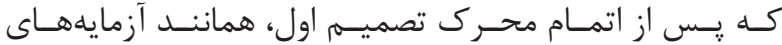

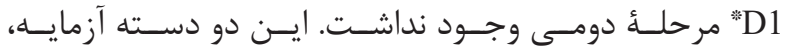

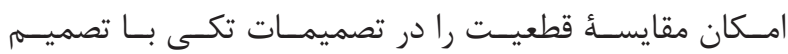

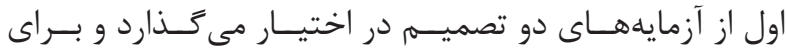

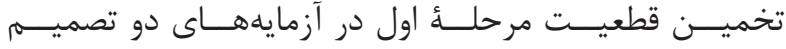
اسـتفاده خواهنــد شــد.

در هــر آزمايـه، قــدرت محــرك (تعـداد نقــاط متحركىى كـهـ

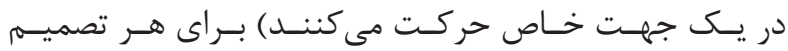

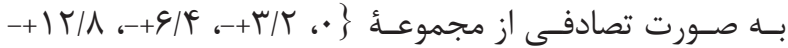
،

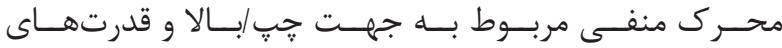

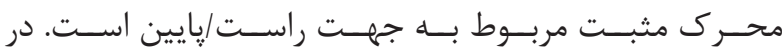

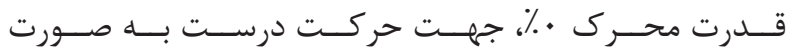

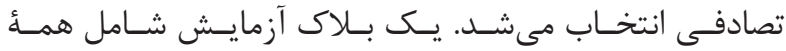

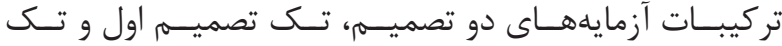

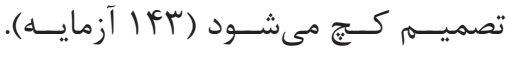

$$
\text { آناليزها و تحليل هاى آمارى }
$$

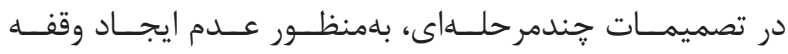

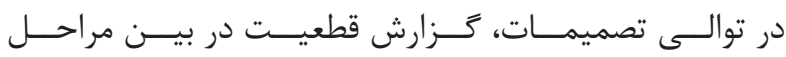

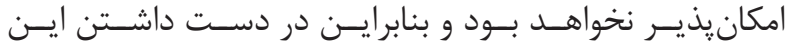

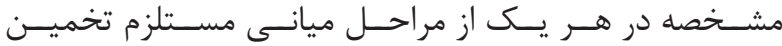

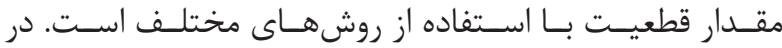

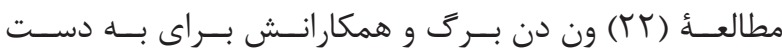

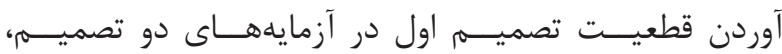

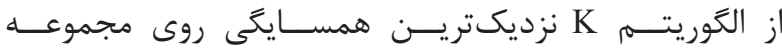

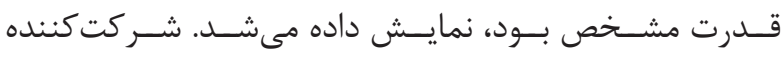

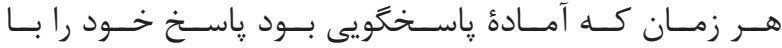

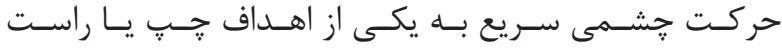

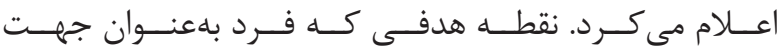

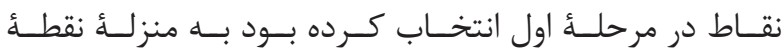

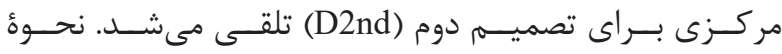

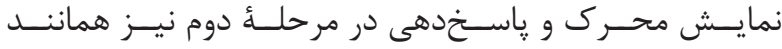

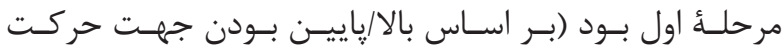

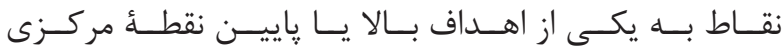

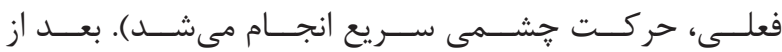

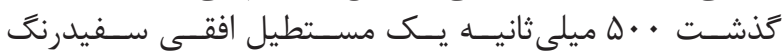

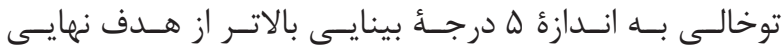

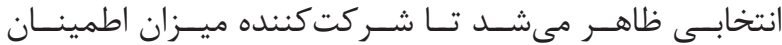

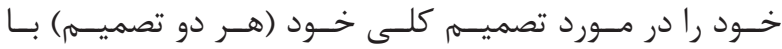

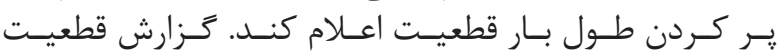

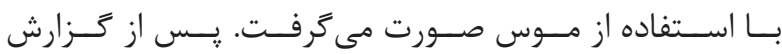

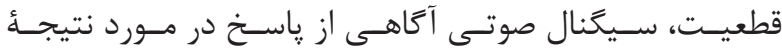

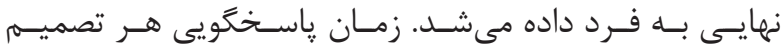

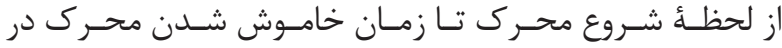

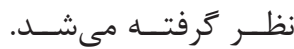

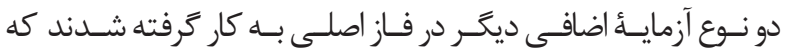

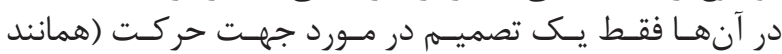

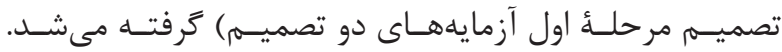

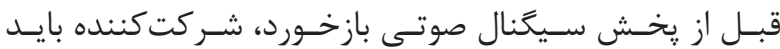

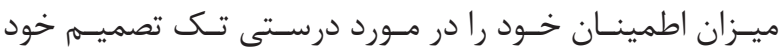

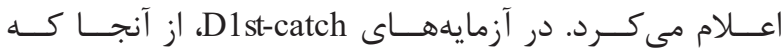

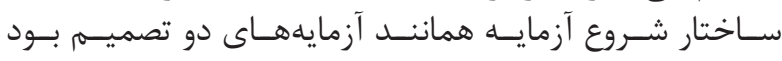

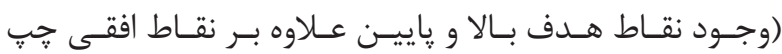
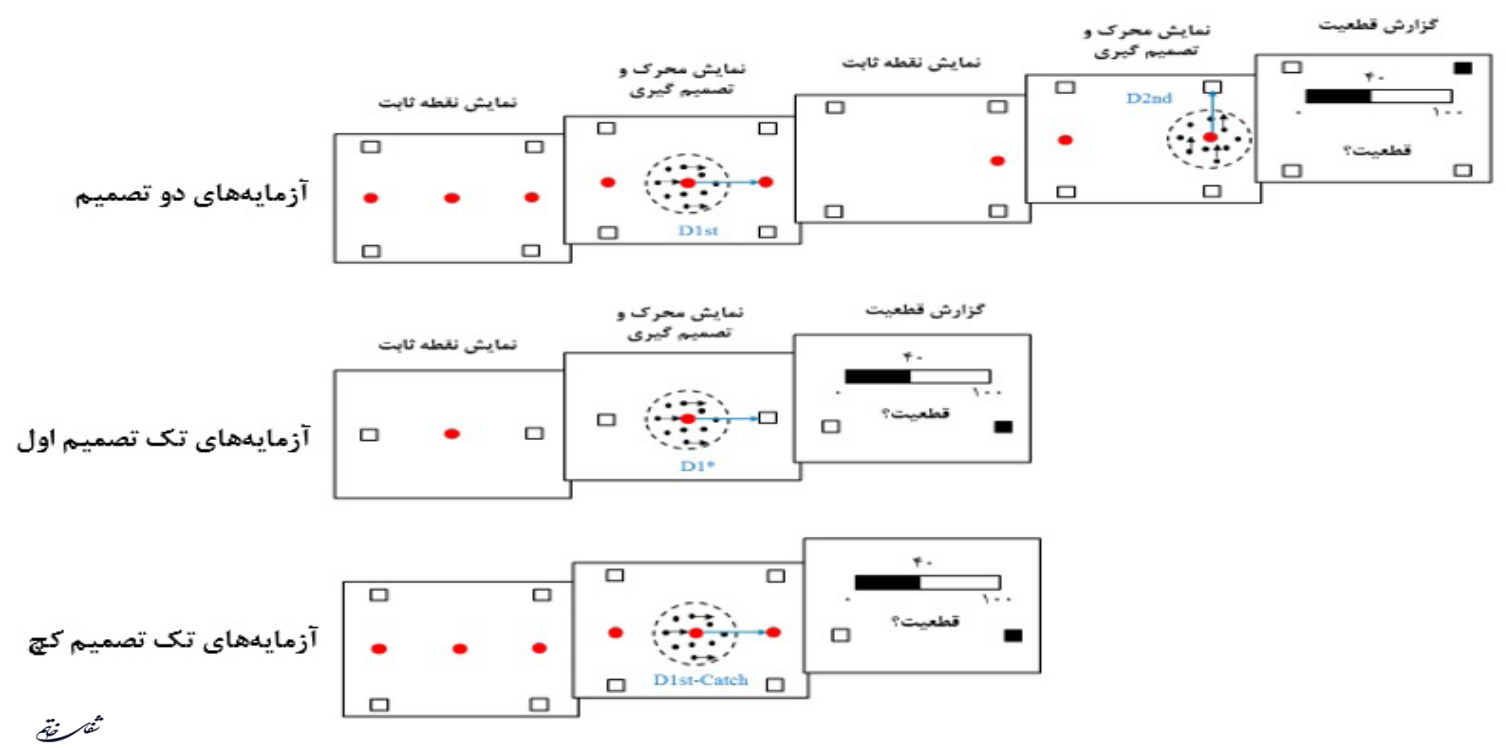

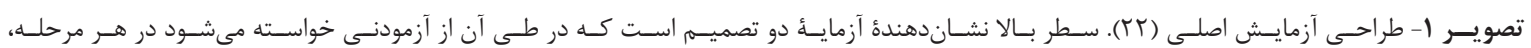

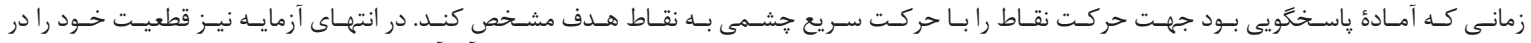

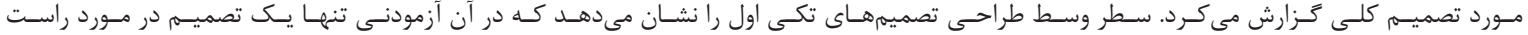

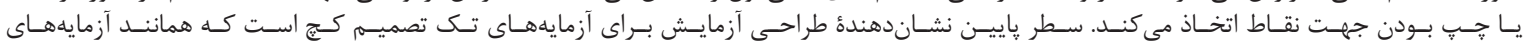

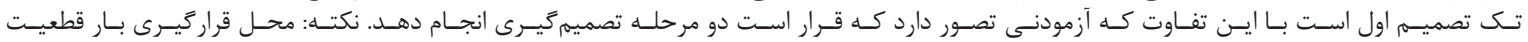


آزمايههــاى D1 و D1st-catch اسـتفاده كردنـد. بديـن شـيوه يافتهها

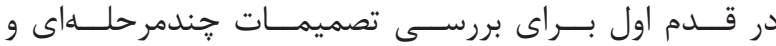

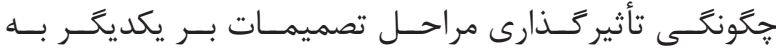

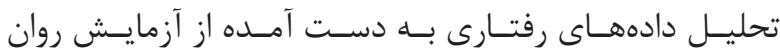

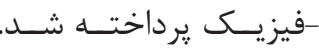

نمــودار ا قسـمت الـف و ب، بـهـ ترتيـب رابطـــهُ دقت و زمــان

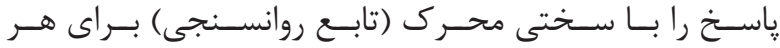

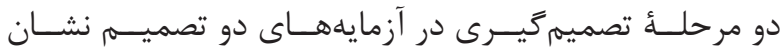

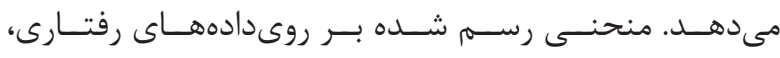

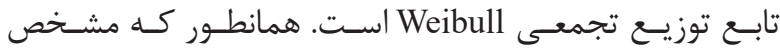

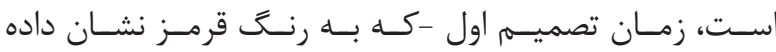

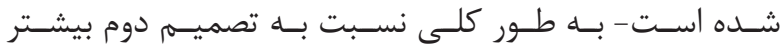

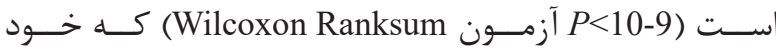

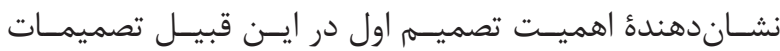

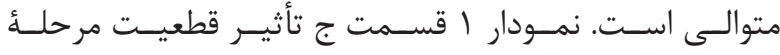

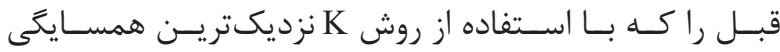

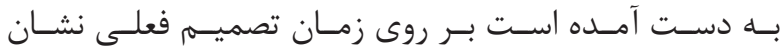

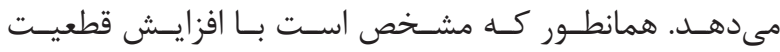

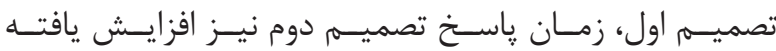

اسـت.

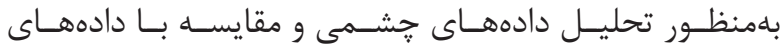

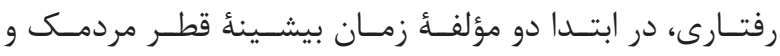

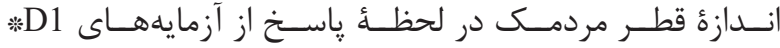

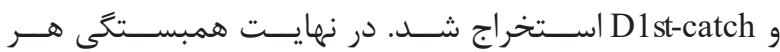

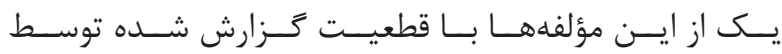

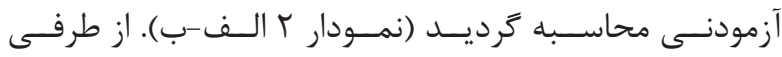

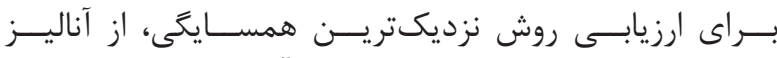
leave one out

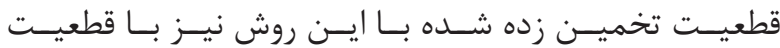

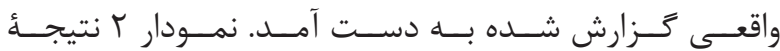

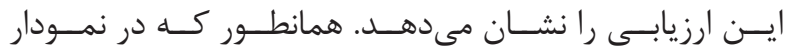

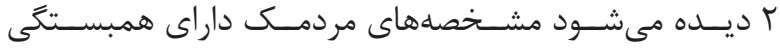

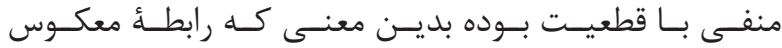

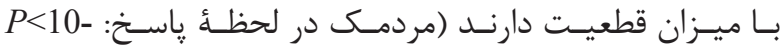

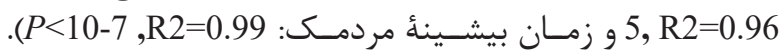

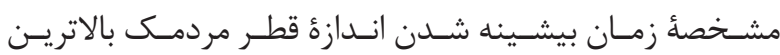

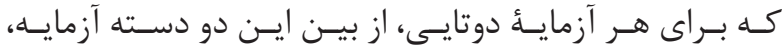

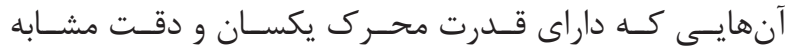

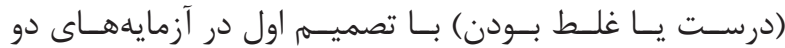

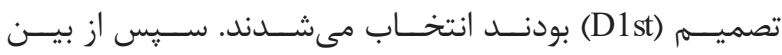

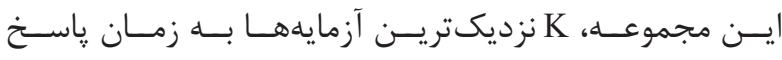

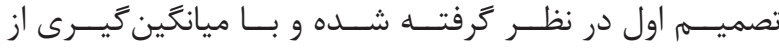

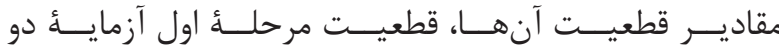

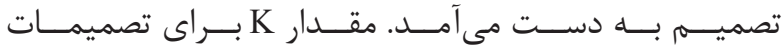

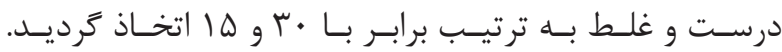

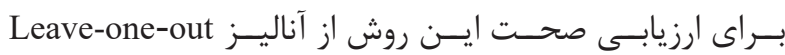

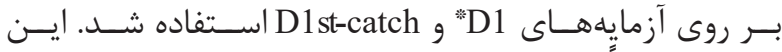

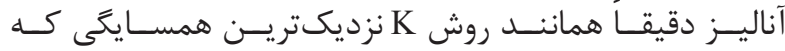

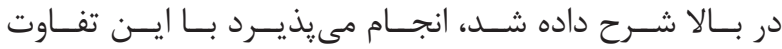

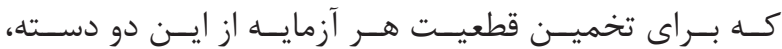

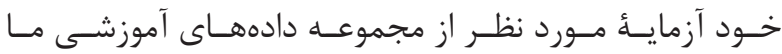

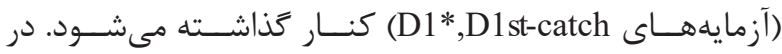

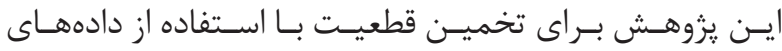

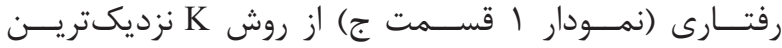

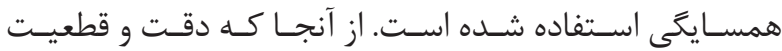

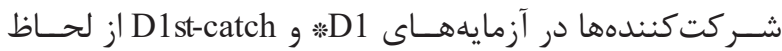
معنــى دارى متفــاوت نبـود (دقـت

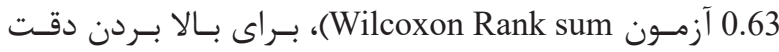

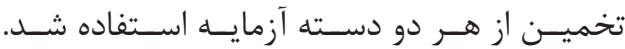

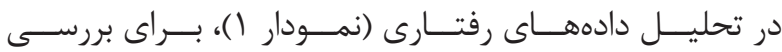

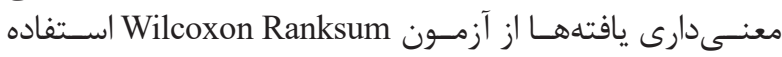

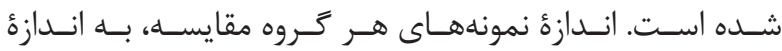

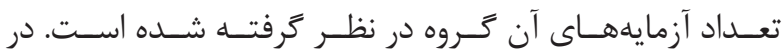

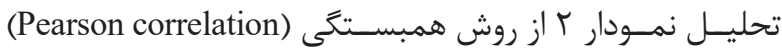

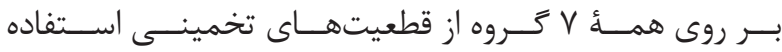

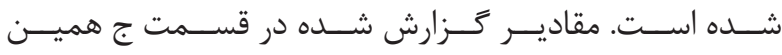

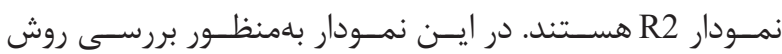

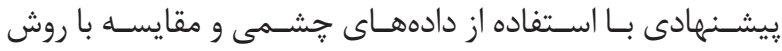

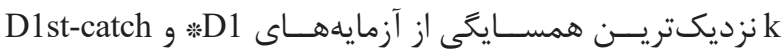

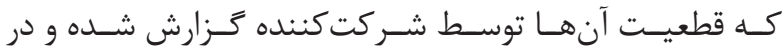

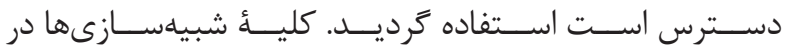

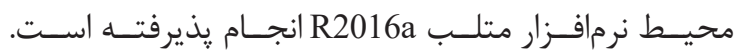

飞

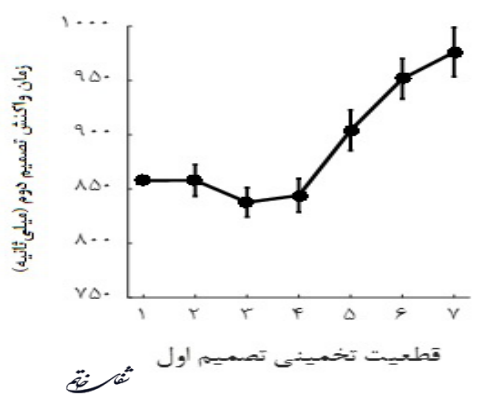

ب

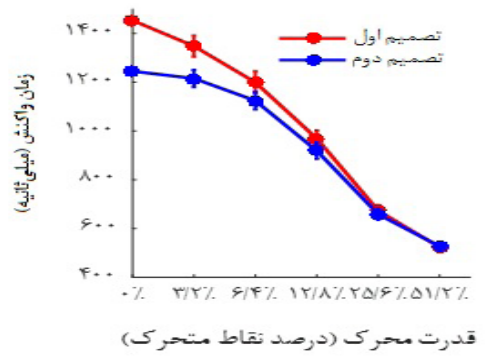

الف

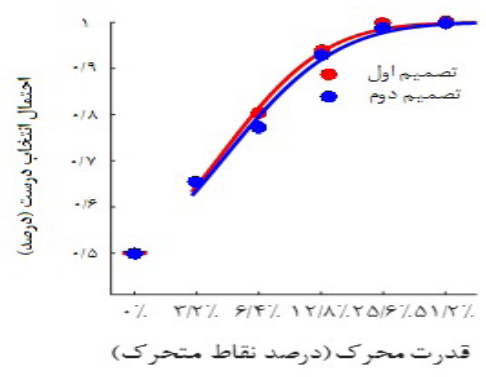

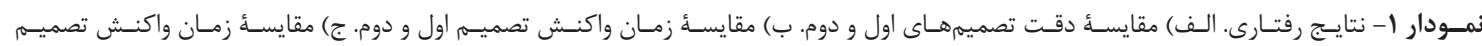

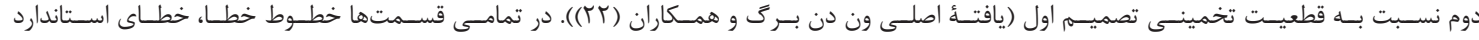




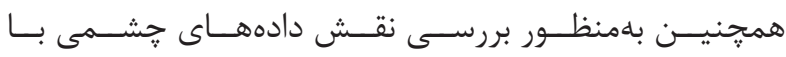

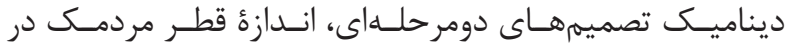

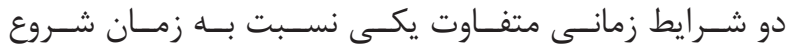

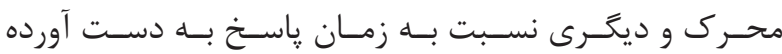

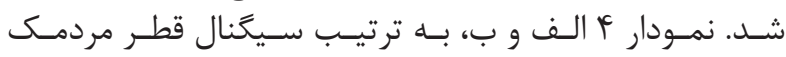

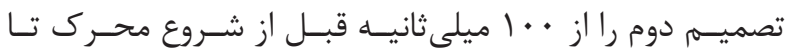

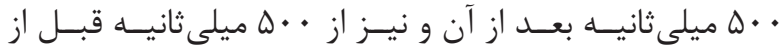

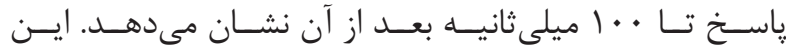

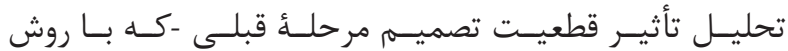

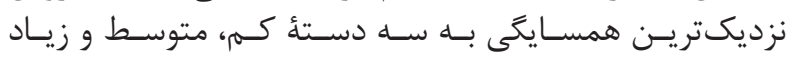

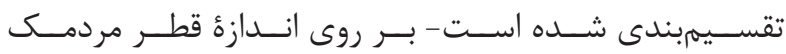

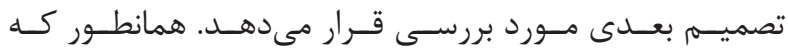

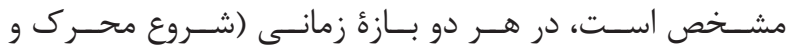

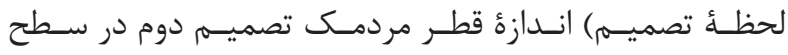

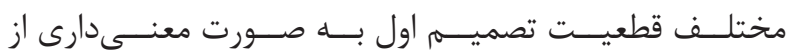

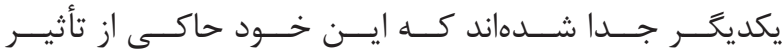

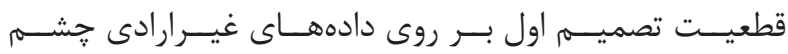

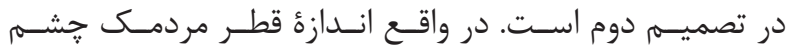

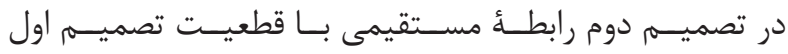

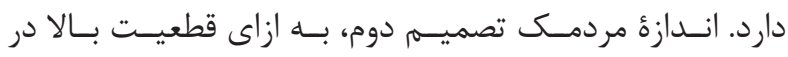

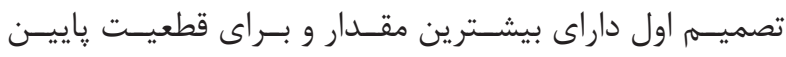

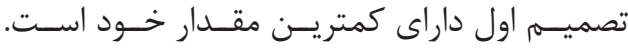

ז

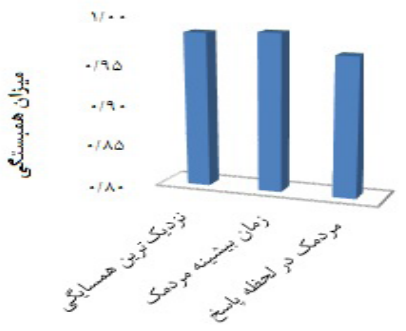

这

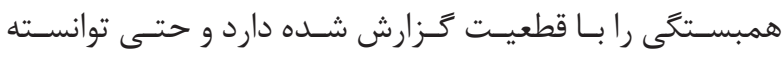

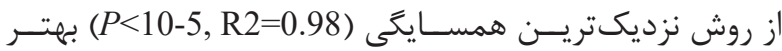

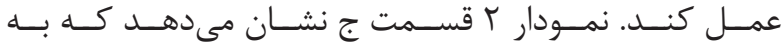

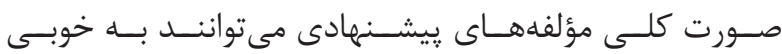

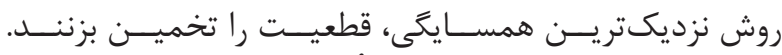

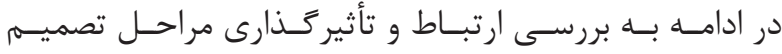

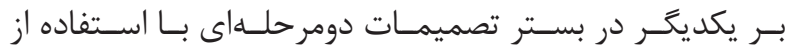

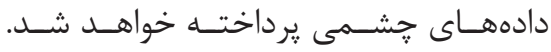

نمـودار ץ ارتبـاط مسـتقيمم قطعيـت تخمينـى تصميـهم اول بـا

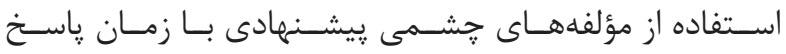

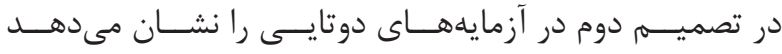

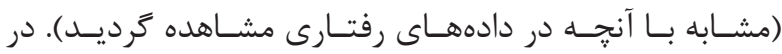

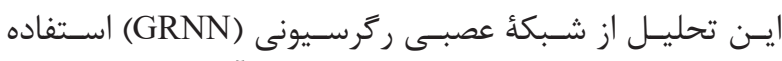

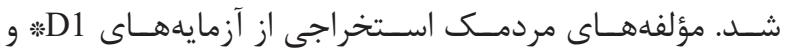

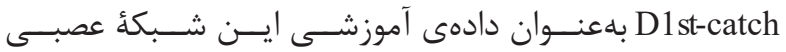

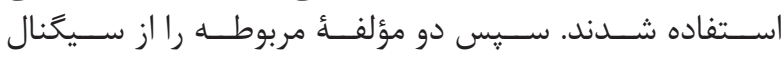

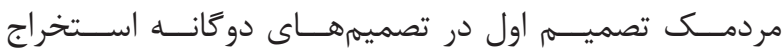

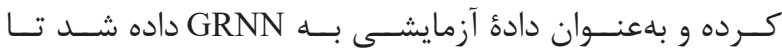

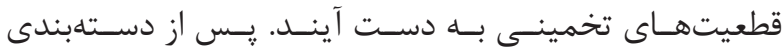

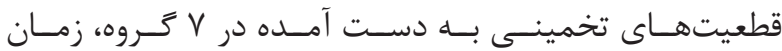

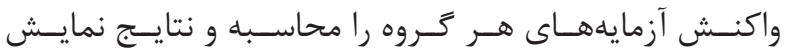

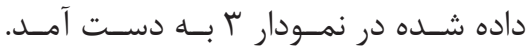

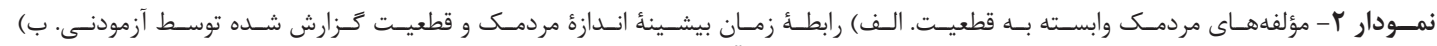

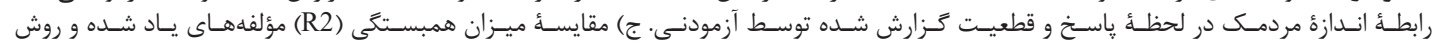
نزديكتريسن همسـايكى.

(ب)

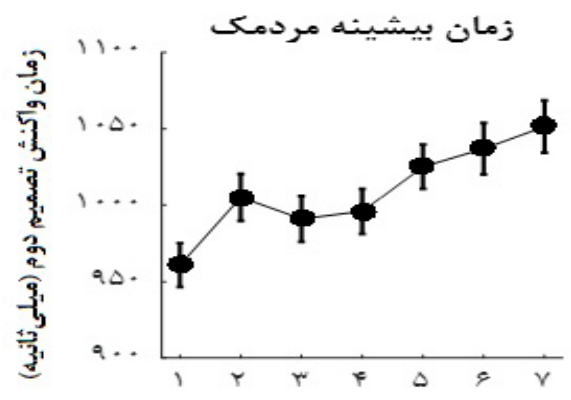

قطعيت تخمينى تصميمى اول
الف)

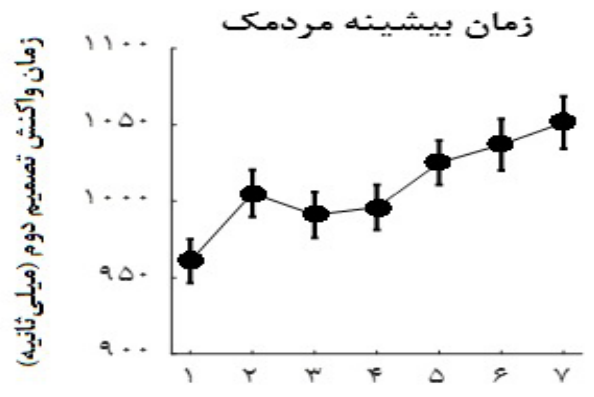

قطعيت تخمينـى تصميمم اول

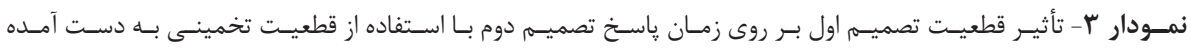

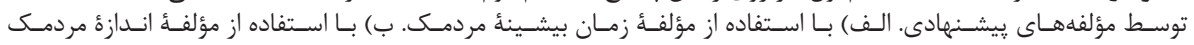

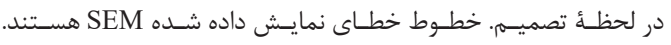


$(ب$

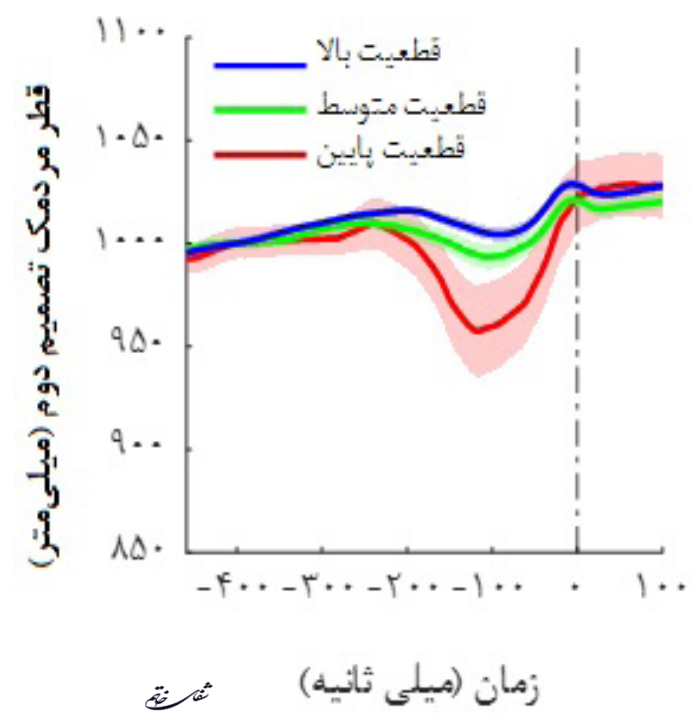

(الف)

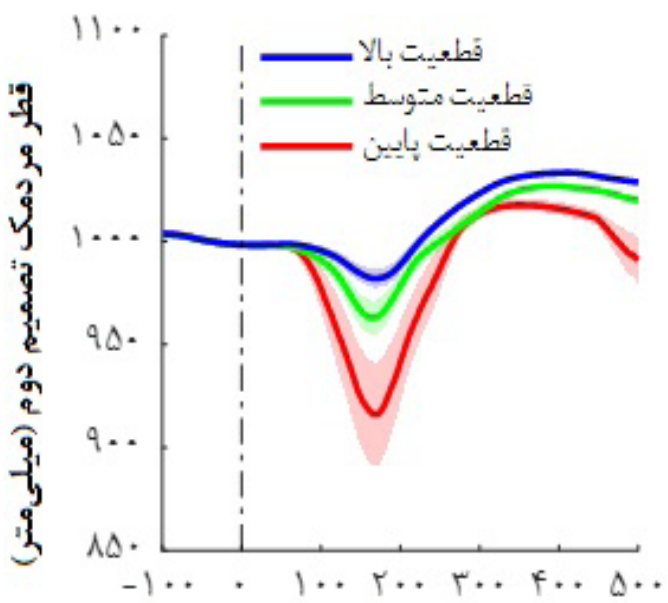

زمان (ميلى ثانية)

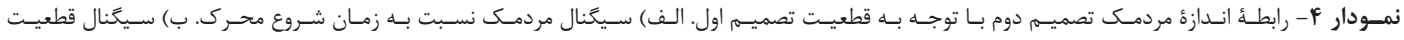

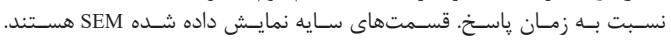

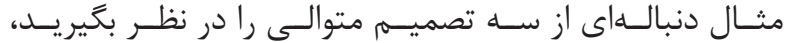

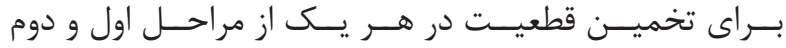

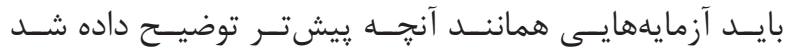

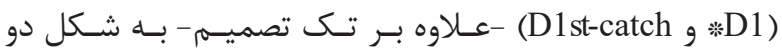

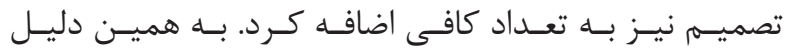

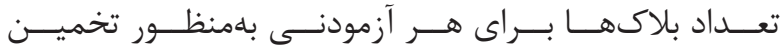

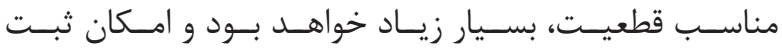

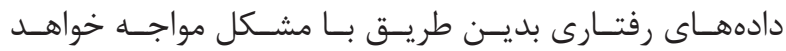

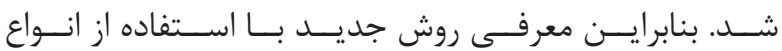

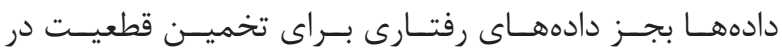

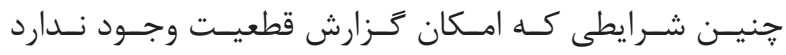

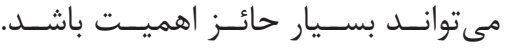

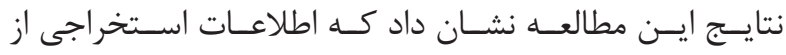

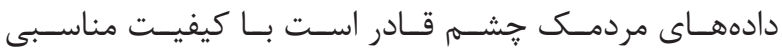

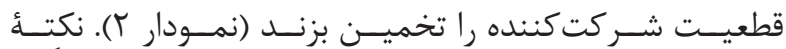

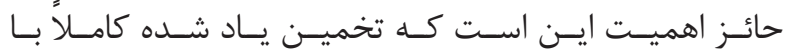

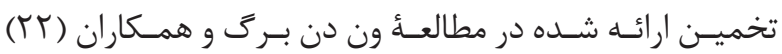

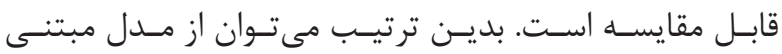

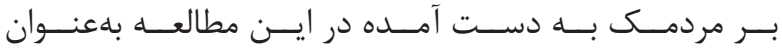

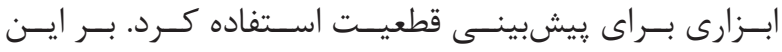

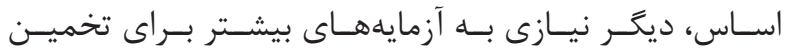

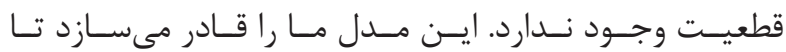

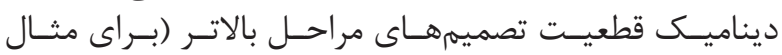

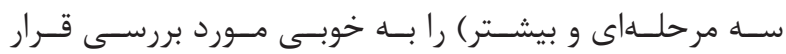

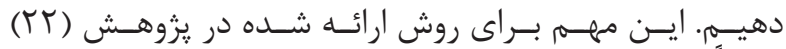

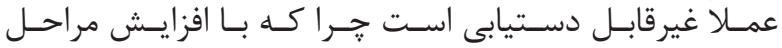

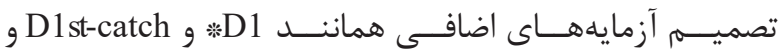

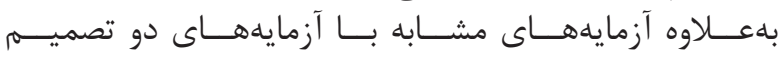

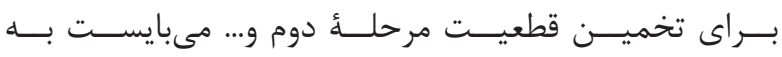

بحث و نتيجه كيرى

نتايسـج دادههـاى رفتـارى نشـان داد در تصميمهــاى تصاى

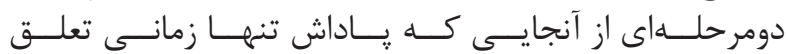

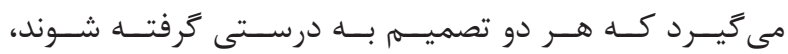

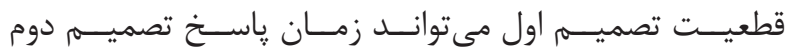

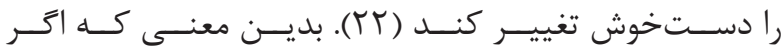

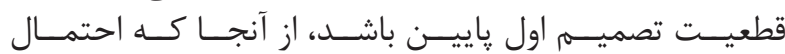

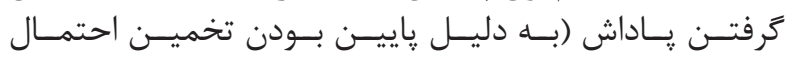

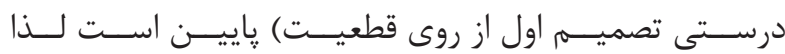

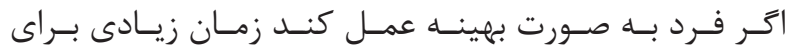

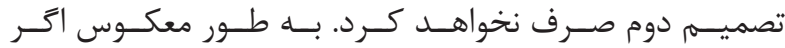

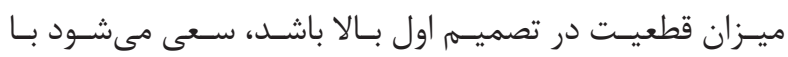

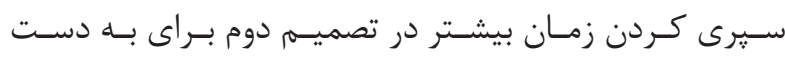

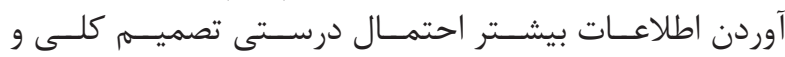

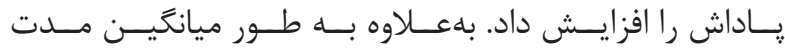

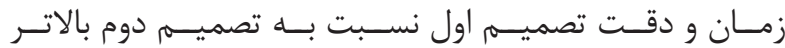

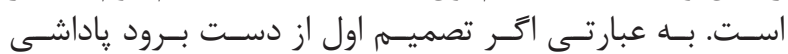

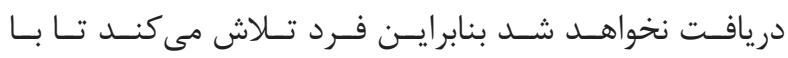

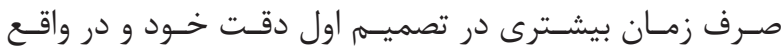

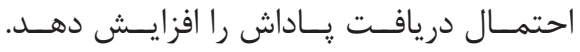

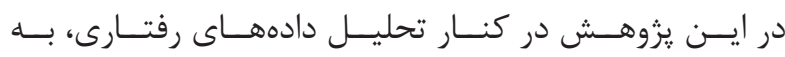

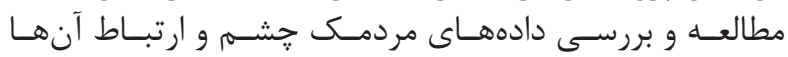

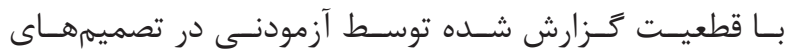

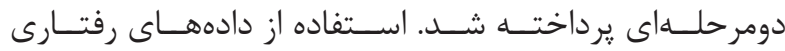

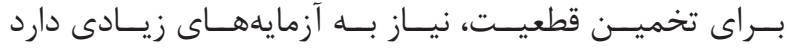

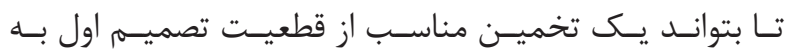

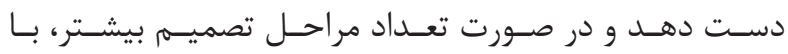

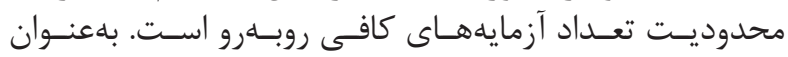




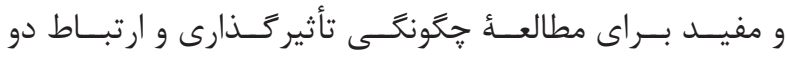

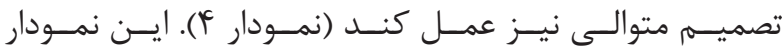

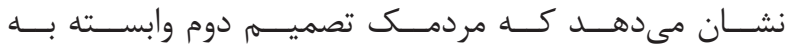

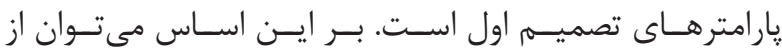

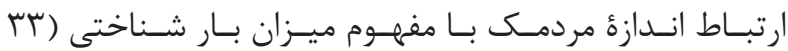

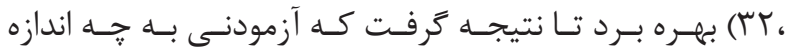

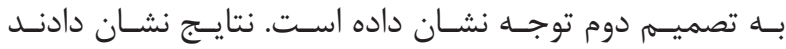

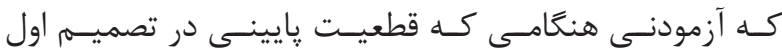

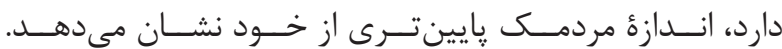

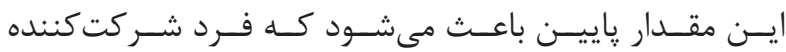

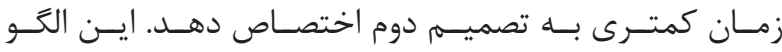

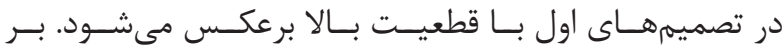

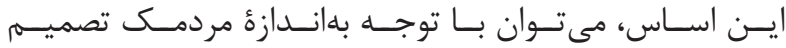

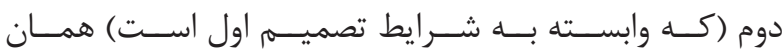

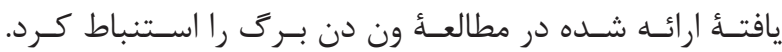

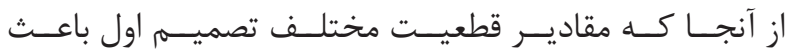

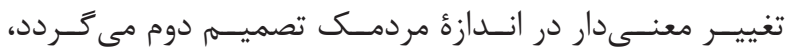

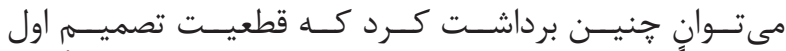

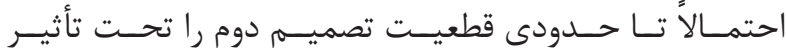

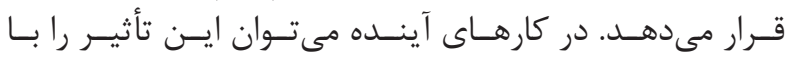

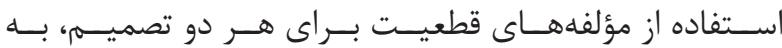
صــورت دقيقتــر مــورد مطالعـه قـــرار داد. تشكر و قدردانى

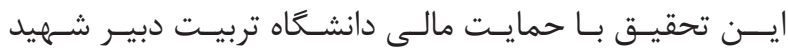

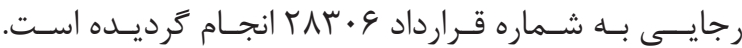

1. Britten KH, Newsome WT, Shadlen MN, Celebrini S, Movshon JA. A relationship between behavioral choice and the visual responses of neurons in macaque MT. Vis Neurosci. 1996; 13(1): 87-100.

2. Esch T, Mesce KA, Kristan WB. Evidence for sequential decision making in the medicinal leech. J Neurosci. 2002; 22(24): 11045-54.

3. Hanks TD, Summerfield C. Perceptual decision making in rodents, monkeys, and humans. Neuron. 2017; 93(1): 15-31.

4. Gold JI, Shadlen MN. The neural basis of decision making. Annu Rev Neurosci. 2007; 30(1): 535-74.

\section{Kiani R, Hanks TD, Shadlen MN. Bounded integration}

in parietal cortex underlies decisions even when viewing duration is dictated by the environment. J Neurosci. 2008; 28(12): 3017-29.

6. Roitman JD, Shadlen MN. Response of neurons in

$$
\text { صـورت تصاعـدى افزايـش ييـدا كنـــد. }
$$

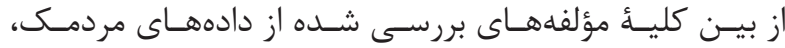

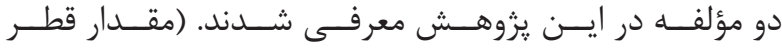

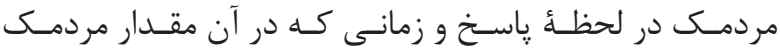

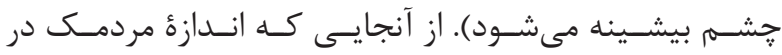

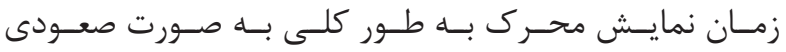

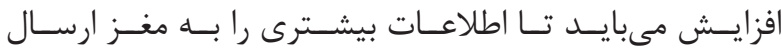

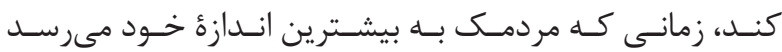

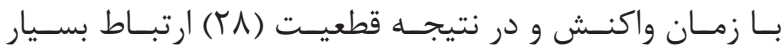

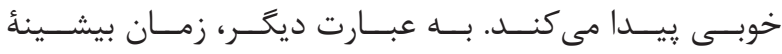

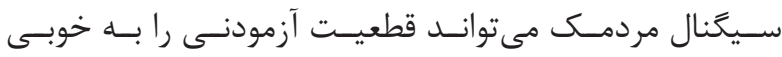

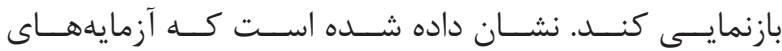

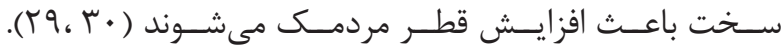

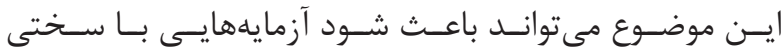

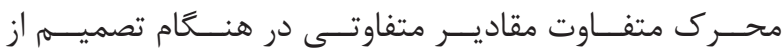

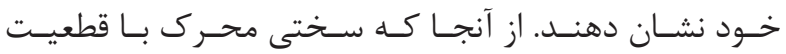

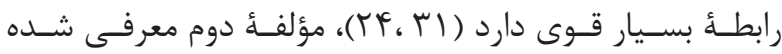

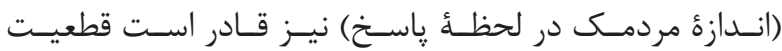

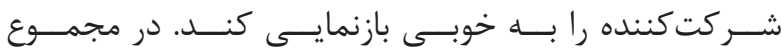

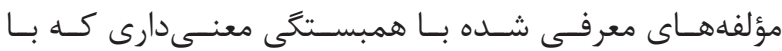

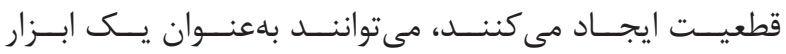

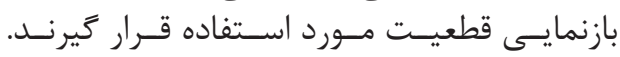

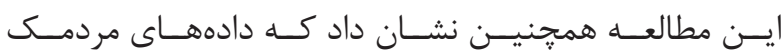

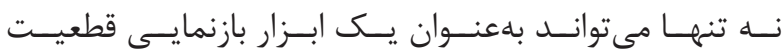

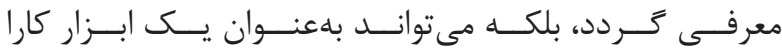

منابع

the lateral intraparietal area during a combined visual discrimination reaction time task. J Neurosci. 2002; 22(21): 9475-89.

7. Kelly SP, O'Connell RG. Internal and external influences on the rate of sensory evidence accumulation in the human brain. J Neurosci. 2013; 33(50): 19434-41.

8. Lorteije JA, Zylberberg A, Ouellette BG, De Zeeuw CI, Sigman M, Roelfsema PR. The formation of hierarchical decisions in the visual cortex. Neuron. 2015; 87(6): 1344-56.

9. Loughnane GM, Newman DP, Tamang S, Kelly SP, O'Connell RG. Antagonistic interactions between microsaccades and evidence accumulation processes during decision formation. J Neurosci. 2018; 38(9): 2163-76.

10. Ratcliff R, Rouder J. Modeling response times for decisions between two choices. Psychological Science. 1998; 9(5): 347-56. 
11. Shadlen MN, Newsome WT. Neural basis of a perceptual decision in the parietal cortex (area LIP) of the rhesus monkey. J Neurophysiol. 2001; 86(4): 191636 .

12. Churchland AK, Kiani R, Shadlen MN. Decisionmaking with multiple alternatives. Nat Neurosci. 2008; 11(6): 693-702.

13. Hanks TD, Mazurek ME, Kiani R, Hopp E, Shadlen $\mathrm{MN}$. Elapsed decision time affects the weighting of prior probability in a perceptual decision task. J Neurosci. 2011; 31(17): 6339-52.

14. Purcell BA, Kiani R. Neural mechanisms of post-error adjustments of decision policy in parietal cortex. Neuron. 2016; 89(3): 658-71.

15. Zizlsperger L, Sauvigny T, Händel B, Haarmeier T. Cortical representations of confidence in a visual perceptual decision. Nature Communications. 2014; 5: 3940 .

16. Purcell BA, Kiani R. Hierarchical decision processes that operate over distinct timescales underlie choice and changes in strategy. Scientific Reports. 2016; 113(31): E4531-E40.

17. Otto TU, Mamassian P. Noise and correlations in parallel perceptual decision making. Current Biology. 2012; 22(15): 1391-6.

18. Kiani R, Churchland AK, Shadlen MN. Integration of direction cues is invariant to the temporal gap between them. J Neurosci. 2013; 33(42): 16483-9.

19. Tohidi-Moghaddam M, Zabbah S, Ebrahimpour $\mathrm{R}$. The role of the primary information on importance of the last information in decision making. Shefaye Khatam. 2016; 4(4): 26-34.

20. Hayden BY, Pearson JM, Platt ML. Neuronal basis of sequential foraging decisions in a patchy environment. Nat Neurosci. 2011; 14(7): 933-9.

21. Olianezhad F, Zabbah S, Ebrahimpour R. The influence of pas $\mathrm{t}$ decision information on decision making in the present. Shefaye Khatam. 2016; 4(3): 1-8.

22. Van den Berg R, Zylberberg A, Kiani R, Shadlen
$\mathrm{MN}$, Wolpert DM. Confidence is the bridge between multi-stage decisions. Current Biology. 2016; 26(23): 3157-68.

23. Fetsch CR, Kiani R, Newsome WT, Shadlen MN. Effects of cortical microstimulation on confidence in a perceptual decision. Neuron. 2014; 83(4): 797-804.

24. Kiani R, Shadlen MN. Representation of confidence associated with a decision by neurons in the parietal cortex. Science. 2009; 324(5928): 759-64.

25. Lempert KM, Chen YL, Fleming SM. Relating pupil dilation and metacognitive confidence during auditory decision-making. PLoS One. 2015; 10(5): e0126588.

26. Strauch C, Greiter L, Huckauf A. Pupil dilation but not microsaccade rate robustly reveals decision formation. Scientific Reports. 2018; 8(1): 13165.

27. Vafaei Shooshtari S, Esmaily Sadrabadi J, Azizi Z, Ebrahimpour R. Confidence representation of perceptual decision by eeg and eye data in a random dot motion task. Neuroscience. 2019; 406: 510-27.

28. Kiani R, Corthell L, Shadlen MN. Choice certainty is informed by both evidence and decision time. Neuron. 2014; 84(6): 1329-42.

29. Brunyé TT, Eddy MD, Mercan E, Allison KH, Weaver DL, Elmore JG. Pupil diameter changes reflect difficulty and diagnostic accuracy during medical image interpretation. BMC Med Inform Decis Mak. 2016; 16(1): 77 .

30. Urai AE, Braun A, Donner TH. Pupil-linked arousal is driven by decision uncertainty and alters serial choice bias. Nature Communications. 2017; 8(1): 14637.

31. Baranski JV, Petrusic WM. Probing the locus of confidence judgments: experiments on the time to determine confidence. J Exp Psychol Hum Percept Perform. 1998; 24(3): 929-45.

32. Chiew KS, Braver TS. Temporal dynamics of motivation-cognitive control interactions revealed by high-resolution pupillometry. Front Psychol. 2013; 4: 15. doi: $\quad 10.3389 /$ fpsyg.2013.00015.

33. Krejtz K, Duchowski AT, Niedzielska A, Biele C, Krejtz I. Eye tracking cognitive load using pupil diameter and microsaccades with fixed gaze. PloS One. 2018; 13(9): e0203629. 